\title{
Ethics outside, within, or beyond OR models?
}

\author{
Marc Le Menestrel $^{1}$ \& Luk N. Van Wassenhove ${ }^{2}$ \\ May 2002 \\ Paper prepared for the Special Issue of Ethics and Operational Research. \\ European Journal of Operational Research.
}

\begin{abstract}
Since ethical concerns are calling for more attention within Operational Research, we present three approaches to combine Operational Research models with ethics. Our intention is to clarify the trade-offs faced by the OR community, in particular the tension between the scientific legitimacy of OR models (ethics outside OR models) and the integration of ethics within models (ethics within OR models). Presenting and discussing an approach that combines OR models with the process of OR (ethics beyond OR models), we suggest rigorous ways to express the relation between ethics and OR models. As our work is exploratory, we are trying to avoid a dogmatic attitude and call for further research. We argue that there are interesting avenues for research at the theoretical, methodological and applied levels and that the OR community can contribute to an innovative, constructive and responsible social dialogue about its ethics.
\end{abstract}

Keywords: Ethics; Models; Processes; OR

JEL Classification: A13, C00, C60

${ }^{1}$ University Pompeu Fabra, Department of Economics and Business, Barcelona, Spain. Marc.Lemenestrel@econ.upf.es.

${ }^{2}$ INSEAD, Fontainebleau, France. Luk.Van-wassenhove@insead.edu. 


\section{Introduction:}

We present three methodological approaches to combine ethics with Operational Research. The first one is ethics outside OR models. It considers that OR models should be objective and that the integration of subjective or ethical values weaken the power and the scientific legitimacy of OR. The second approach is ethics within OR models. It considers that the objectivity of OR models is illusory, ill-suited to the social nature of the OR application domain, and that it may constitute a denial of responsibility not to attempt to include subjective and ethical values within OR models. The third approach is ethics beyond OR models. It considers that whatever the model, there remain subjective and ethical values beyond it. OR then consists in balancing OR models with the process of OR. It emphasizes the role of social dialogue.

Our motivation for this caricature is twofold. First, we are not always comfortable with possible consequences of integrating too much subjectivity within the core methodology of OR. Although these attempts are full of good intentions, we believe that OR legitimacy may be at risk since good intentions may not always apply once these models are developed and used at a large scale. Second, we believe that this opposition between objectivity and subjectivity may be more than a figure of speech. We suggest for instance how it may constitute a meta-model with innovating properties. Ethics beyond OR models could lead to a methodological approach that links OR models to a rational and open discourse on the limitations of these models.

At a time when ethical concerns seem to grow among OR researchers, we would contribute if we clarify some of the fundamental and difficult trade-offs that our community must face and discuss. On the other hand, we are optimistic about the finding of a rationale in the combination of OR with ethical concerns, as well as about the effectiveness of concrete institutional and individual efforts to combine OR models with an ethical practice. 


\section{Ethics outside OR Models}

Traditionally, models used in Operational Research have aimed at identifying an optimal solution independent of the perceptions, appreciation and feelings of the human beings. When this is the case, these models identify an "objectively optimal" solution and any subjective value is abstracted. Objective models of Operational Research exclude ethical concerns in order to ensure the formal validity of their solution.

These objective models, like all models based on a maximization principle, inherit from models of the natural sciences (For a history of OR/MS, see Corbett and Van Wassenhove 1996; for the link with natural sciences, see Brans 2002, and for the maximization principle, see Sen 1997). The natural sciences methodology aims at understanding things independently of subjective values and provides OR models with a scientific legitimacy. In natural science like in objective OR models, the ideal is universal truth.

Not only are there no ethical concerns properly included within objective OR models, there should be no ethical concerns within them. Otherwise, they would not be objective anymore and would lose their scientific legitimacy. In an excellent book about ethics and models, Wallace reports a large consensus of this position:

"One of the ethical responsibilities that was agreed upon is that the goal of any model building process is objectivity with clear assumptions, reproducible results, and no advocacy" (Wallace, 1994, p. 6)

This ideal position must be qualified. It has long been recognized that models of natural sciences do not capture the entire real world. Modeling embeds a process of abstraction that leaves out singular properties of real situations. This process is not totally objective and is value-loaded. An honest effort from the model builder to make the model as objective as possible is then presented as a deontological responsibility:

"Participants generally agree on some aspects of ethical conduct, such as the need for model builders to be honest, to represent reality as faithfully as possible in their models, to use accurate data, to represent the results of the model as clearly as 
possible, and to make clear to the model user what the model can do and what its limitations are." (Wallace, 1994, p. 8)

If OR models are not perfectly objective, their ideal is to remain as objective as possible, and this does not exclude deontological responsibilities of the model builder. In principle, there is no incompatibility between "ethics outside OR models" and the statement that OR actors should follow ethical rules or codes of conduct.

The critic of objective models is paradoxically methodological. The argument resides in the nature of the social world as opposed to the natural world that inspired objective OR models. Brans (2002, p. 342) recalls that:

"The main feature of the modeling of Natural Real World situations is that the Physical Law remains unchanged and is universally disseminated".

Human values, whatever they may be, are evolving and are locally specific. Therefore, applying a Natural World modeling to the social world amounts to the abstraction of the singularities of human beings, of societies, and of their evolutions. In the social world, these human values are meaningful. They influence actual decisions and therefore should be part of the methodological approach.

Hence, the main criticism is that objective OR models are based on a conception of scientific methodology that does not properly reflect the specificity of social sciences with respect to natural sciences, i.e. the fact that human beings have values which are evolving and are local. There is a fundamental methodological inability of objective OR models to integrate these specific concerns.

When we act in a social world, is it really ethical to abstract from social values? Does this not amount to ignore ethics or to placing ethics at an inferior level, allegedly less legitimate? By relegating human values outside the realm of scientific methodology, objective OR models weaken the legitimacy of human values. Beyond the methodological question thus resides an ethical one.

These ethical issues cannot be addressed by referring solely to the objectivity of OR models. The claim of objectivity would appear as a denial of responsibility. Since objectivity does not guarantee ethics, why not include ethics within OR models? What would it mean to include ethical values within OR models? Would it be a guarantee of a methodology more respectful of human values? 


\section{Ethics within OR models}

The integration of subjective concerns within OR models received a powerful tool around the 1970s with the development of multi-criteria approaches (MCDA). Although they are not the unique way to integrate ethics within OR models, we illustrate our argument with this type of models (see Brans, 2002; Keeney and Raiffa, 1976; Roy and Bouyssou, 1993). In these models, there is no one single objective function but several. How these functions combine (their respective weights), depends on parameter values that are not determined by the objective functions themselves. In this sense, the optimal solution depends on "subjective" weights and we hence call these models "subjective".

Theoretically, this distinction between objective and subjective can be elusive. For a given set of weights, and with appropriate conditions on the combination of the different attributes, the method amounts to the maximization of a function, be it composed of several underlying functions or not. Indeed, the distinction in this case is more of a practical nature. The need to measure the weights induces de facto difficult measurement issues and an acknowledgement of the specificity of the situation at hand. In this manner, a relationship between the model builder and the model user is established. This relationship, which tended to be abstracted in the scientific methodology of objective models, becomes integrated within the scientific methodology of subjective OR models.

But the distinction can be theoretical too, for instance when subjective OR models relax some necessary assumptions of objective models in an attempt to reflect the difficulty of measuring the weights (say by using intervals). For instance, in the Prometheus-Gaïa model, only intervals within which weights are deemed to remain are measured and plugged in the model. For a given set of weights, these models do not necessarily identify an optimal solution and when one is identified, it may not be unique or attainable. Such a subjective model is theoretically "weaker" than objective models. In practice, these models constitute an "aid" to decision-making rather than an absolute criterion of rationality (e.g. Brans, 2002).

In the face of the growing interest for ethical concerns, the flexibility of these models has led Professor Brans to propose that ethics could be treated as a specific set of weights. While subjective weights would reflect subjective values of individuals, 
ethical weights would reflect values at the level of society or humanity and in particular sustainable development. Hence, this approach distinguishes a form of "individual subjectivity" (the subjectivity of the decision-maker or the model builder) from a "social subjectivity" that could be integrated within OR models to render them more ethical. This allows Brans to balance objective, subjective and ethical concerns ${ }^{3}$. Some solutions can be optimal both from the subjective point of view and from the ethical point of view. Otherwise, an ethical conflict arises, modeled by the incompatibility of the two sets of weights.

Practically, ethical weights may be even more difficult to measure than individually subjective weights. Brans proposes to have the weights determined by a group of independent experts. This raises many questions: who then is going to choose these experts? How are they going to agree on a set of weights? Is it realistic to assume that any group of experts can ever measure ethical values to the point of constituting a set of weights to be plugged in an OR model? Indeed, should we succeed in forming such a committee, would it be the best use we could make of it? Eventually, we may end up integrating ethical concerns within OR models by outsourcing their measurement, which is the key of their influence. Like for individually subjective considerations, integrating ethical concerns within OR models assumes somehow a "good intention" on the part of the decision-maker, the analyst and the (ethical) experts. Even with these models, considerations of an ethical nature remain essentially outside of the model. Crucial elements are 1) the ability to properly measure the subjective weights (individual), 2) the ability to have the ethical weights properly measured (experts group), 3) the integration of these weights into the objective core of the model, 4) the interpretation of the model when it does not identify an optimal solution without ambiguity, 5) the good faith of all actors.

The assumption of good faith is of particular importance in the case of subjective models. With subjective OR models, it is easier to construct a model that legitimizes ex-post the selection of a solution identified ex-ante. The distortion of the

${ }^{3}$ Brans (2002) calls "rational" what we call "objective" but with essentially the same meaning. For reasons obvious in section 4, we want to avoid the reduction of rationality to objective rationality. 
model takes place in the choice of the weights and is difficult to detect. For someone else than the decision-maker and the builder of the model, it may be impossible to appreciate a possible manipulation. In the wrong hands, subjective models can be strategic instruments to legitimize decisions through a distortion of scientific methodology. From the point of view of objectivity, subjective models raise an important question as to the scientific credibility of OR. We believe this trade-off to be of great importance in the debate about ethics outside or within OR models. Furthermore, this trade-off between objectivity and subjectivity may be an object of scientific study in itself. This is discussed in the next section.

\section{Ethics beyond OR models}

On one hand, excluding ethics from OR models is in itself an ethical position and on the other hand, including ethics within OR models excludes some ethical questions. Whatever the model is, i.e. whether it includes ethical considerations or not, there will always remain ethical issues beyond the model. Is there a way to combine a model with the ethical concerns that are necessarily beyond it? This is the approach we term ethics beyond OR models.

One way to explore such an approach is to acknowledge the practical dimension of OR (Fortuin, van Beek and Van Wassenhove, 1996). OR is not reduced to its models and their analytical way to look at things. Therefore, it can be taken for granted that "all models are incomplete" and that many considerations remain outside of any model (Little, 1994, p. 179). One possibility to express this embedding of OR models within a practical context consists in treating OR as the conjunction of a model together with the relation between this model and reality (Figure 1). We argue below that this approach to OR allows us to reflect a synthesis of the concepts evoked in the preceding sections. 


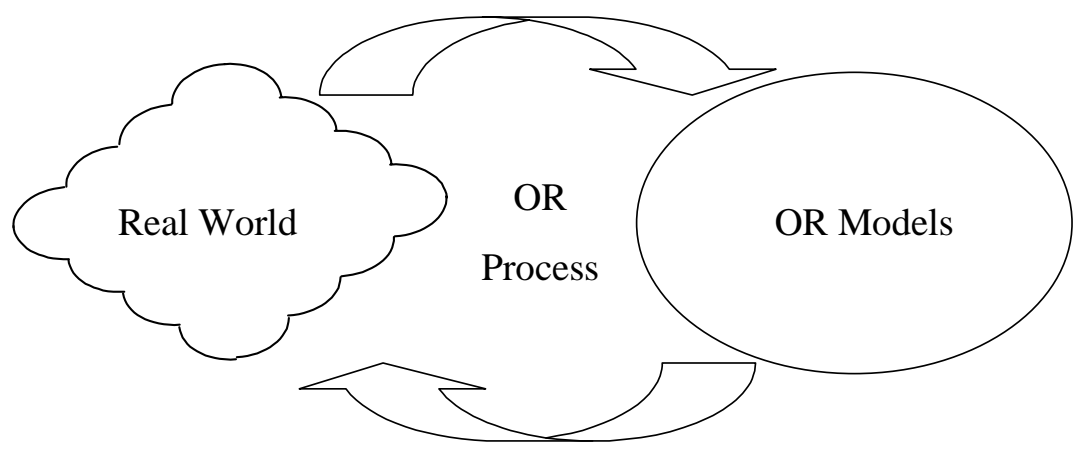

Figure 1: OR combining OR models and OR Process

By separating OR models from the real world, we make explicit the relation between them. This relation, called the "OR process", is a specific entity that is neither within the model nor part of the real world. The process of OR is independent from the real world as well as beyond the model.

In an earlier and exploratory methodological paper (Le Menestrel \& Van Wassenhove, 2001), we have suggested to relax the assumption that this relation between a model and reality is invariant. In other words, for a given model and in a given real situation, there may be many ways to relate the model with the reality (in the words of Sen (1986), we relax "procedural invariance"). Further, these processes may be valued in themselves as part of the practice of OR and independently of the valuation of the different solutions identified by the model. Not only the result counts but also the way it has been obtained and will be applied in practice.

For example, we evoked in section 2 a deontological responsibility of the model builder with regard to the limitations of a model and thus referred to an ethical valuation of the process of OR. In an objective approach that treats ethics outside OR models, such a procedural valuation should be minimized and only the valuation of the solutions within the models bears practical significance. In a subjective approach that treats ethics within OR models, one attempts to embed a procedural valuation within the substance of the model itself by broadening it. In both cases, the procedural nature of this valuation is not treated specifically nor systematically. In objective and subjective approaches to OR models, the intrinsic value of the process of OR is not an integral part of the methodology. We suggest that there may be a heuristic power in separating OR models from the process of OR and in studying their complementary combination. 
We have argued that two fundamental types of values are of importance in OR as part of the social sciences. Values founded in regularities and expressed by laws and values that are idiosyncratic to the situation at hand (Carrier and Wallace 1994). This finds a natural formulation in the distinction between OR models and OR process: OR models reflect the regularities and measure them; on the other hand, OR processes are subject to an idiosyncratic valuation, specific to the situation at hand. In other words, OR models correspond to the theoretical side of OR which attempts to construct a measure as objective as possible of the different solutions that are modeled. The OR process corresponds to the practical side of OR whose valuation necessarily remains subjective because it is not captured in the model.

Of course, this separation between objective and subjective is relative. If, for a given model, we can define the singularities outside of it as "all the properties of the real world that are abstracted by the model", some of these singularities may well be included in another broader model. A property of the real world is not objective or subjective per se: it depends on the model that is built to reflect it. The distinction remains nevertheless relevant for all models. There will be characteristics of the real world that will be modeled as regularities and others that will be left out as singularities. Therefore, the combination of procedural, practical, idiosyncratic, subjective values with substantive, theoretical, regularities-based, objective values is a relevant issue, wherever the line is to be drawn. When we regroup different characteristics of OR models (theoretical, objectively valued, etc.) to oppose them with the process of OR (practical, subjectively valued, etc.) we are looking for some sort of a "meta-model" that would characterize the methodology of OR as a social science.

This meta-model may well take the form of the combination of a quantitative and a qualitative valuation. Indeed, we may recall that the identification of regularities when building a model has for primary purpose the quantification of the valuation of the different solutions of the model (see Brans, 2002). It is thus natural to consider that the process of OR, because it is not included within the model, remains qualitative. In this sense, this approach beyond OR leads to the combination of a quantitative and a qualitative dimension. A last section now discusses some implications of this suggestion. 


\section{Towards a Theory and an Ethics of Combining Qualities and Quantities}

In ethics beyond OR models, theoretical solutions implemented in practice seem to receive a double valuation. A quantitative valuation that is objectively determined by the model, and a qualitative one that reflects a given and singular point of view and which originates in the practical process of implementing this solution in the real world. When the quantitative measurement and the qualitative valuation coincide, this procedural approach identifies one optimal solution that is both objectively and subjectively optimal. When the quantitative measurement and the qualitative appreciation do not coincide, this procedural approach does not identify a solution that is both objectively and subjectively optimal. It reflects a dilemma. As a trade-off between a quantitative and a qualitative valuation, such a dilemma between an OR model and an OR process may well be a very specific type of trade-off.

Consider the following example. Suppose we can represent the quantitative evaluation of each theoretical solution to an OR model by a quantitative scale: 1, 2, 3, ... ${ }^{4}$ Suppose further that we can represent the qualitative evaluation of each practical implementation of a solution on a qualitative scale: $1^{\text {st }}, 2^{\text {nd }}, 3^{\text {rd }}, \ldots$. Note that the qualitative scale is intuitive to reflect ethical concerns, but ethical concerns or part of them may be also modeled within the quantitative scale, as discussed in section 3. We want to minimize the quantitative valuation (it may, for instance, represent costs) and favor the first ones of the qualitative valuation. Then, a solution with valuations (1, $\left.1^{\text {st }}\right)$ is clearly optimal. It is better on the quantitative and qualitative sides $(1<2$ and $\left.1^{\text {st }}>2^{\text {nd }}\right)$. However, a situation may well oppose a solution with valuations $\left(1,2^{\text {nd }}\right)$ and a solution with valuations $\left(2,1^{\text {st }}\right)$. In such a case, the trade-off is irreducible and the meta-model does not identify a solution that is optimal on both theoretical (OR model) and practical grounds (OR process). Therefore, even though all solutions are valued quantitatively as well as qualitatively, the combination of these valuations does not necessarily lead to the identification of an optimal solution. Observe that it is not

\footnotetext{
${ }^{4}$ We will illustrate our argument with a ratio scale and an ordinal scale to make precise the distinction between quantitative and qualitative (see Le Menestrel \& Van Wassenhove, 2001).
} 
the objective model that is rendered more flexible through the consideration of subjective qualitative valuations within it. It is its combination with a qualitative valuation beyond it that renders the whole approach flexible. The natural conjunction of the two types of valuations in the optimal case guarantees nevertheless that the approach does not become arbitrary. A procedural approach to reflect the specificity of each point of view on a given model may thus lead to innovative theoretical research. This idea of combining a quantitative scale with a qualitative scale deserves, we believe, more theoretical research.

From a more practical point of view, this approach suggests that analysts can adopt an objective approach to OR models while still being able to give subjective and ethical concerns the methodological place they deserve. Instead of looking for a quantification of these concerns, the methodology would aim at making them explicit through a discursive approach. In the words of Barabba (1994):

"Only through open discussion of the questions can model assumptions and limitations - as well as values - be revealed and assessed."

Indeed, the need for close and on-going communication between the model builder and user was emphasized by Barraba (1994) and Little (1994). But we should make it explicitly part of the methodology, and there are good reasons for this. Indeed, the OR analyst has direct experience of the abstraction process that takes place when constructing the model. Therefore, he should play a leading role in rendering explicit the aspects of the situation that are not taken into account by the model. In this manner, the OR analyst provides useful elements for subjective valuation beyond the model. Further, given a qualitative valuation, he can make explicit its combination with the quantitative evaluation of the model and identify dilemmas or optimal solutions. The principle that the model should remain as objective as possible offers a common measure for the discursive confrontation of the different points of view. This would not be the case in a purely qualitative discursive situation without any model to rely on. In a sense, we are trying to build on communicational ethics as a bridge between theoretical and practical dimensions of human behavior (Habermas, 1992). This is why we believe that keeping objective and subjective considerations separated may help to combine OR with ethics. 
We posit that our suggested approach is more realistic in order for OR methodology to provide ethics the place it deserves. Suppose a group of experts is formed to provide an independent ethical opinion on an energy choice for a business actor. The analyst may have constructed a model to identify the solution that minimizes costs. He may even have used a model to identify and quantify the environmental impact of each solution. How should he rely on the expert group to balance these two objectives? Would assessing relative weights for the expert group guarantee an ethical decision? It may be more efficient to present the model(s), as objectively as possible, as well as all the assumptions and the limitations already identified. An open discourse could then allow the experts to point at some inherent methodological limitations. For instance, for some solutions, we may not be able to calculate the full environmental consequences, not even on a probabilistic basis. A discursive approach would allow the experts to emit an opinion about the relevance of conducting further research before acting in this case (precaution principle). ${ }^{5}$ An effective discursive process would allow considerations that are not taken into account by the model to be made explicit and valued, even qualitatively. Finally, the participants could propose a qualitative ordering of the solutions according to their respect of the environment. Having such a consensus explicit or even public could help the decision-maker to manage the trade-off between his goals and ethics. As the actor, he would bear the responsibility of his choice. In this discursive approach, the outcome is an explicit acknowledgment of the values beyond the model and qualitative valuation process that does not weaken the objectivity of the model.

\section{Conclusion}

We have presented three approaches to ethics and Operational Research: ethics outside, within and beyond OR models. We have emphasized their distinctions but

5 This emphasis on discursive approach to address complex, irreversible, long term, uncertain environmental issues is advocated by the Intergovernmental Panel on Climate Change report (2001, Chapter 10: Decision-Making Frameworks). An example has been the European Commission process to prepare for the negotiation of the Kyoto protocol (see van den Hove, 2000). 
also their complementary character, trying to avoid a dogmatic approach that would claim to be "the best" in all circumstances.

Our approach suggests some interesting avenues for research, in order to further clarify the assumptions of the three approaches, but also, and more specifically, to characterize the interaction between OR models and OR process. There are interesting theoretical issues in combining quantitative and qualitative scales.

There is clearly a need for real-life case studies comparing the three different approaches and showing their strengths and limitations. Since this touches upon social sciences, we should learn empirically, by examples, if we wish to progress. Similarly, we may learn from other disciplines (philosophy, ethics) and professions (engineering, law, medicine) that have developed codes of ethical conduct long ago and may show some similarities (engineering is model based but also applied to social contexts as in man-machine interface design for instance). We may need to learn to be more sensitive to cultural differences since ethics is about values that can be specific to the individuals and society concerned.

There are also strong implications of the three approaches on the role and behavior of the actors of OR: the analyst, the professional societies, etc. (see e.g. Leet and Wallace, 1994). Consider analysts taking an oath of ethical conduct and being backed up by their professional society when they disclose non-ethical use of OR. Obviously, this would have big implications for the analysts, the society and the user or decision-maker (see for instance Fienberg, 1994).

Ethical issues of Operational Research clearly deserve further work of theoretical, methodological and applied nature. We should be pro-active in our OR community instead of waiting until being "Enroned"...or being put in Andersen's situation.

\section{References}

Barabba, V.P., 1994. The Role of Models in Managerial Decision-Making Never Say the Model Says. In: Wallace, W.A. (Ed), Ethics in Modeling, Elsevier Science Ltd., Oxford UK, pp. 145-160.

Brans, J.P., 2002. Ethics and Decision. European Journal of Operational Research 136, 340-352. 
Carrier H.D., Wallace, W.A., 1994. An Epistemological View of Decision Aid Technology with Emphasis on Expert Systems. In: Wallace, W.A. (Ed), Ethics in Modeling, Elsevier Science Ltd., Oxford UK, pp. 37-57.

Corbett and Van Wassenhove, 1996. Crisis? What Crisis? Four Decades of Debate on Operational Research. In: Fortuin, van Beek and Van Wassenhove, 1996. OR at wORk: Practical experiences of operational research. Taylor \& Francis, London, pp 289-304.

Fienberg, S.E., 1994. Ethical and Modelling Considerations in Correting the Results of the 1990 Decennial Census. In: Wallace, W.A. (Ed), Ethics in Modeling, Elsevier Science Ltd., Oxford UK, pp. 103-144.

Fortuin, van Beek and Van Wassenhove, 1996. OR at wORk: Practical experiences of operational research. Taylor \& Francis, London.

Habermas, J. 1992. Moral Consciousness and Communicative Action (Studies in Contemporary German Social Thought). Boston MA: MIT Press.

Keeney, R. L. and H. Raiffa: 1976, Decisions with Multiple Objectives: Preferences and Value Tradeoffs, Wiley, New York.

Intergovernmental Panel on Climate Change, 2001. Climate Change 2001: Mitigation. Available at http://www.ipcc.ch/.

Le Menestrel, M., Van Wassenhove, L., 2001. The Domain and Interpretation of Utility Functions: An Exploration. Theory and Decision, 51, Forthcoming.

Leet, E.H., Wallace, W.A., 1994. Society's Role in the Ethics of Modeling. In: Wallace, W.A. (Ed), Ethics in Modeling, Elsevier Science Ltd., Oxford UK, pp. 242246.

Little, J.D.C., 1994. On Model Building. In: Wallace, W.A. (Ed), Ethics in Modeling, Elsevier Science Ltd., Oxford UK, pp. 167-183.

Roy, B., Bouyssou, D., 1993. Aide multicritère à la Décision : Méthodes et Cas. Economica, Paris.

Sen, A., 1986. Information and invariance in normative choice. In: Social Choice and Public Decision Making, Cambridge University Press, pp. 29-55.

Sen, A., 1997. Maximization and the Act of Choice, Econometrica 65: 745-779. 
van den Hove, S., 2000. Participatory approaches to environmental policymaking: the European Commission Climate Policy Process as a case study. Ecological Economics 33, 457-472.

Wallace, W.A. (Ed.), 1994. Ethics in Modeling. Elsevier Science Ltd., Oxford UK. 